\title{
Study on catalytic removal of toluene by metal oxides derived from hydrotalcite
}

\author{
Liu Hongbo ${ }^{1}$, Huang Zhiyong ${ }^{1, *}$ \\ ${ }^{1}$ School of Materials Science and Environment, Beijing Institute of Technology Zhuhai, 519088 Zhuhai, China
}

\begin{abstract}
Hydrotalcite (HT) precursor was synthesized by coprecipitation method, and the surface of HT precursor was modified. The mixed metal oxide (MO) catalyst was prepared by calcination of HT precursor, which was used to remove toluene from VOCs assisted by NTP technology. The catalytic performance of MO catalyst was investigated. The results show that the MO catalyst with good structure can be obtained after calcination of HT precursor before and after modification. The results of catalytic performance test showed that the initial concentration of toluene was $700 \mathrm{ppm}$, the gas flow rate was $600 \mathrm{~mL} / \mathrm{min}$, and the reaction time was $30 \mathrm{~min}$, SIE of NTP was above $3.0 \mathrm{~kJ} / \mathrm{L}$, the toluene conversion rate reached above $90 \%$. MO assisted NTP had better catalytic performance with lower energy consumption. The toluene treatment capacity per unit energy consumption increased from $15.3 \%$ to $20.6 \%$, which had an increase of $34.6 \%$.
\end{abstract}

\section{Introduction}

According to the definition of the World Health Organization (WHO), volatile organic compounds (VOCs) refer to the volatile organic compounds with saturated vapour pressure over $133.322 \mathrm{~Pa}$ and boiling point between $50{ }^{\circ} \mathrm{C}$ and $260{ }^{\circ} \mathrm{C}$ at room temperature. VOCs have wide sources and carcinogenic effect on human $[1,2]$. In addition, VOCs react with nitrogen oxides, hydrocarbons and oxidants in the atmosphere under sunlight to produce photochemical smog, which is harmful to human health and crop growth. Common VOCs control technologies can be divided into two categories. One is non-destructive technology, that is, VOCs are separated from the exhaust gas by absorption or condensation. The other one is called destructive technology, that is, the technology to degrade VOCs into $\mathrm{CO}_{2}$ and $\mathrm{H}_{2} \mathrm{O}$ by combustion method, biodegradation method, catalytic oxidation method and low temperature plasma technology (NTP) [3]. In recent years, plasma technology for the treatment of various pollutants has become one of the hot topics [4]. The active components of catalysts for VOCs combustion can be divided into noble metal oxides and non-noble metal oxides. Among them, non-noble metal oxide catalysts mainly include perovskite, spinel and composite oxide catalysts. These catalysts are relatively low in price and also show good catalytic performance. Their disadvantages are relatively low catalytic activity and high ignition temperature [5]. In view of the problems of hard control of structure and poor selectivity of metal oxide catalysts, hydrotalcite was used as the precursor to prepare mixed metal oxide catalysts for decomposition of VOCs pollutants. The structure and activity of the catalysts can be controlled by adjusting the type and composition of cation and anion properly. At the same time, high catalytic activity can be obtained by using the "synergistic effect" of various metals assisted by NTP. The development of the project has important practical significance and social benefits for effective control of VOCs emissions, environmental protection and promotion of human health. It will provide important experimental data for the research and development of structurally controllable metal oxide catalysts, and will have a wide application prospect in the field of new materials and environmental protection.

\section{Experimental}

\subsection{Synthesis of hydrotalcites}

Hydrotalcite precursors were synthesized by coprecipitation method. The main synthesis steps are as follows: nitrate solution A was prepared according to a certain stoichiometric ratio, and the base solution $\mathrm{B}$ with a certain amount of $\mathrm{NaOH}$ and $\mathrm{Na}_{2} \mathrm{CO}_{3}\left(\left[\mathrm{OH}^{-}\right]=1.6\right.$ $\left.\left(\left[\mathrm{M}^{2+}\right]+\left[\mathrm{M}^{3+}\right]\right), \quad\left[\mathrm{CO}_{3}{ }^{2-}\right]=2.0\left[\mathrm{M}^{3+}\right]\right)$ was prepared. The salt solution A was added into the base solution $\mathrm{B}$ under stirring, and the $\mathrm{pH}$ value was controlled to be about 9 . The slurry $\mathrm{C}$ was transferred into a three-neck flask and stirred vigorously. At a certain temperature, the mixture was crystallized for a certain time. The mixture was filtered and washed to neutral with distilled water. The filter cake is obtained. Distilled water and KH550 was added and stirred at $70{ }^{\circ} \mathrm{C}$ for $1 \mathrm{~h}$ and filtered. The filter cake was dried at $70{ }^{\circ} \mathrm{C}$ in a drying oven for 24 hours, and the experimental flow chart is shown in Figure 1.

\footnotetext{
* Corresponding author: 05066@bitzh.edu.cn
} 


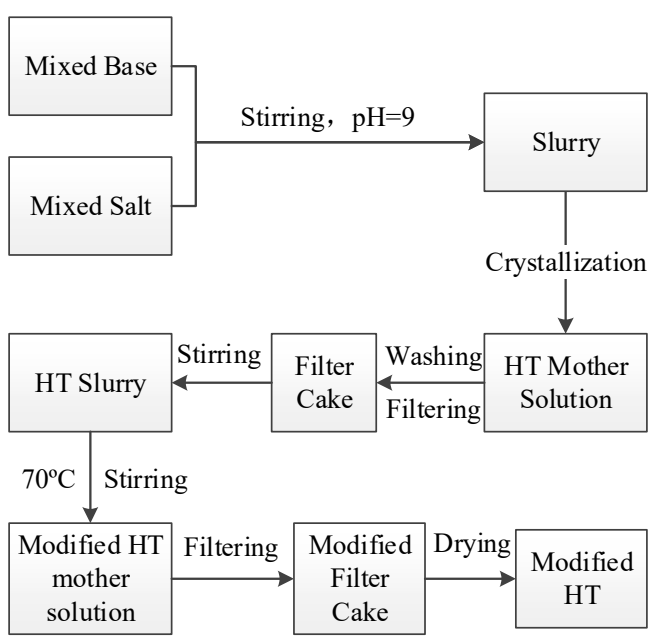

Fig. 1. The experimental flow chart of HT precursor.

\subsection{Characterization of hydrotalcites}

$\mathrm{X}$-ray diffraction (XRD) patterns were determined using a Shimadzu 6100X diffractometer equipped with $\mathrm{Cu} \mathrm{K}$ a radiation $(\lambda=1.5406 \AA$ ) operating with $40 \mathrm{kV}$ and 30 $\mathrm{mA}$.

\subsection{Preparation of mixed metal oxide catalysts}

Mixed metal oxides were prepared by calcination of the obtained HT precursor. The HT precursor was put into muffle furnace, heated from room temperature to $500{ }^{\circ} \mathrm{C}$ with a heating rate of $3{ }^{\circ} \mathrm{C} \cdot \mathrm{min}^{-1}$ in air atmosphere. It was then kept at this temperature for $3 \mathrm{~h}$, and was then cooled down to room temperature.

\subsection{Test of catalytic behaviour}

\subsubsection{Experimental setup}

The experimental setup consists of three parts: gas distribution system, dielectric barrier discharge reactor and gas composition analysis system. The toluene bottle was placed in a $30{ }^{\circ} \mathrm{C}$ constant temperature circulating water bath. The toluene was then mixed with dry air in a buffer bottle, and put into a DBD plasma reactor for treatment. At the same time, toluene automatic monitor are connected to the emission pipeline to monitor the toluene concentration in the emissions online.

\subsubsection{Experimental method}

$50 \mathrm{~mL}$ toluene liquid was first injected when using the above experimental device to treat the waste gas containing toluene. The valve of dry air cylinder was then opened, the intake air flow was adjusted and the initial concentration of toluene $\mathrm{C}_{\text {in }}$ was measured. Then open the plasma generator and adjust the working voltage of the voltage regulator to the required experimental value, and start timing after the plasma discharge is stable. After a certain period of time, the relevant indicators were determined and analysed. The emission was absorbed by deionized water, and the absorption solution was determined to evaluate the concentration of key active species and the acidic components of the emission.

\subsubsection{Determination index and analysis method}

The concentration of toluene in the emission of the reactor were detected by MultiRAE Lite PGM 6208 gas detector. Removal rate of toluene $(\eta, \%)$ is calculated by the following formula:

$$
\eta=\left(\mathrm{C}_{\text {in }}-\mathrm{C}_{\mathrm{out}}\right) / \mathrm{C}_{\mathrm{in}} \times 100 \%
$$

Where: $\mathrm{C}_{\text {in }}$ and $\mathrm{C}_{\text {out }}$ are the concentration of toluene at the inlet and outlet of DBD reactor respectively (ppm).

Specific input energy (SIE) is calculated by the following formula:

$$
\mathrm{SIE}=\text { Power } / \text { Gas flow rate }(\mathrm{J} / \mathrm{L})
$$

The $\mathrm{pH}$ value and conductivity of absorption solution was measured by Shanghai ray magnetic DZB-718 Portable multi parameter analyzer.

\section{Results and discussion}

\subsection{The structure of HT and MO}

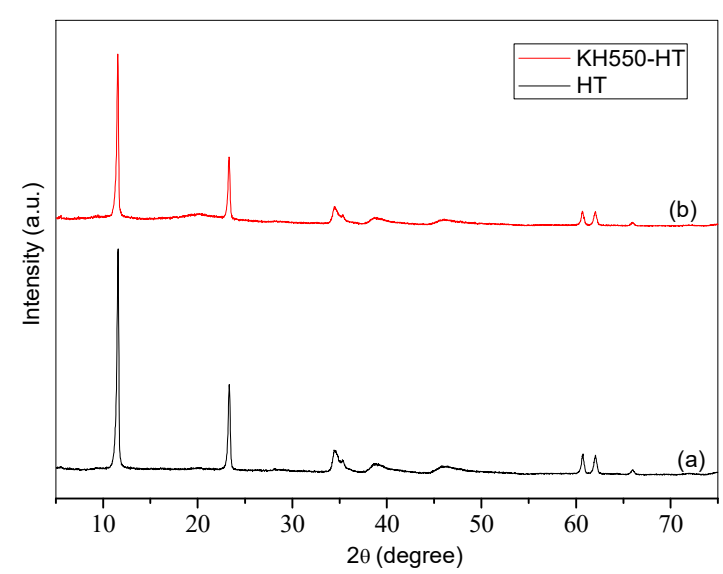

Fig. 2. The XRD pattern of HT before and after the modification of KH550.

XRD patterns of HT precursor prepared before and after modification of KH550 were shown in Figure 2. Both of the samples show similar patterns. Compared with the standard spectrum of HT, it can be found that the samples show the characteristic diffraction peaks of HT. Therefore, the HT precursors with good structure can be synthesized.

XRD patterns of MO catalysts prepared by calcination of HT precursors were shown in Figure 3. It can be seen from the figure that the characteristic diffraction peak of HT precursor has almost disappeared. The MO catalyst shows the diffraction peak of $\mathrm{MgO}$ with stable baseline and wide peak shape. This is consistent with the literature report. It indicates that the 
structure of HT precursor has been destroyed and metal oxides have been transformed. Therefore, metal oxide catalysts were successfully prepared by HT precursors. In addition, the samples before and after modification showed similar XRD patterns, which was consistent with the XRD results of HT precursors.

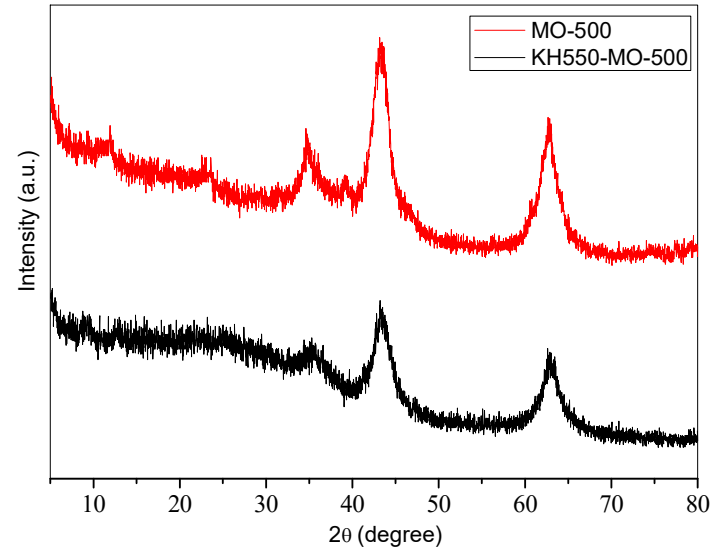

Fig. 3. The XRD patterns of MO prepared by calcination of HT precursors before and after the modification of KH550.

\subsection{The effect of reaction time}

The effect of reaction time was studied. The relationship between reaction time and toluene conversion rate was shown in figure 4 . It can be obtained that with the increase of reaction time, the conversion rate of toluene was increased at first, and was then increased slowly. The conversion rate reached $96.3 \%$ when the reaction time is $30 \mathrm{~min}$. The toluene conversion rate reached $99.1 \%$ when the reaction time increased to $60 \mathrm{~min}$. When the reaction time continued increased, the conversion concentration of toluene was not changed anymore. Therefore, $30 \mathrm{~min}$ was chosen as the reaction time.

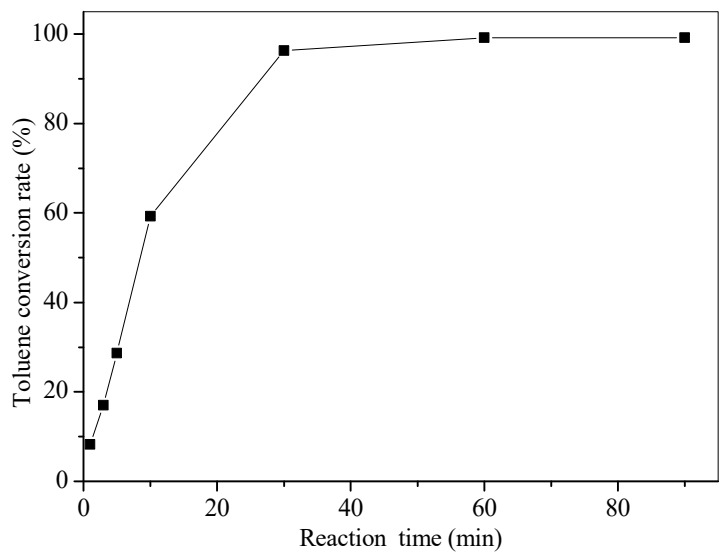

Fig. 4. The relationship between reaction time and toluene conversion rate

\subsection{The effect of initial concentration of toluene}

The effect of initial concentration of toluene was also studied. The relationship between initial concentration of toluene and toluene conversion rate was shown in figure 5. It can be obtained that with the increase of initial concentration of toluene, the conversion rate of toluene was decreased slowly. The toluene conversion rate reached $100 \%$ when the initial concentration was under $200 \mathrm{ppm}$. The toluene conversion rate slowly decreased with the increase of concentration of toluene. It could still keep the toluene conversion rate above $99 \%$ when the initial concentration of toluene reached $600 \mathrm{ppm}$. The toluene conversion rate decreased to $94.6 \%$ when the concentration of toluene reached $900 \mathrm{ppm}$. Therefore, $600 \mathrm{ppm}$ was chosen as the initial toluene concentration.

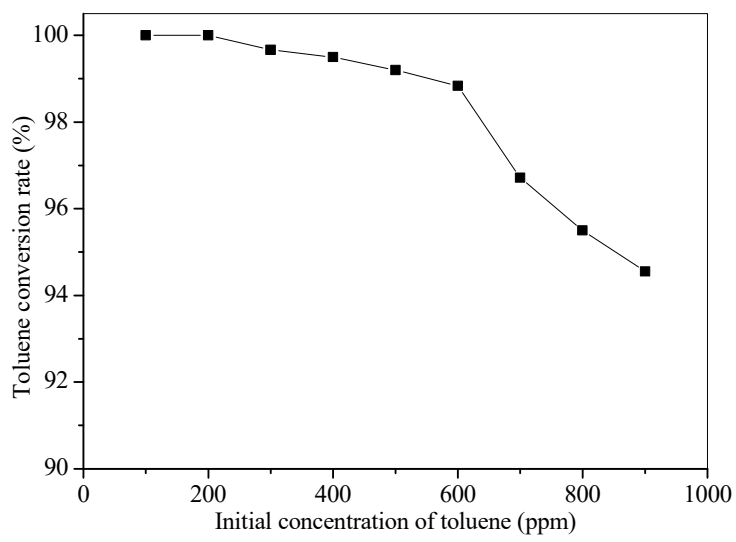

Fig. 5. The relationship between initial concentration of toluene and toluene conversion rate

\subsection{The effect of gas flow rate}

The effect of gas flow rate was investigated in the paper. The relationship between gas flow rate and conversion rate of toluene was shown in figure 6 . The flow rate has effect on toluene conversion rate. The conversion rate of toluene was decreased with the increase of gas flow rate. It has a maximum of $99.7 \%$ when the gas flow rate is $300 \mathrm{~mL} / \mathrm{min}$. It could still keep the toluene conversion rate above $99 \%$ when the gas flow rate of toluene reached $600 \mathrm{~mL} / \mathrm{min}$. The toluene conversion rate is still $93.6 \%$ with the gas flow rate of $1800 \mathrm{~mL} / \mathrm{min}$.

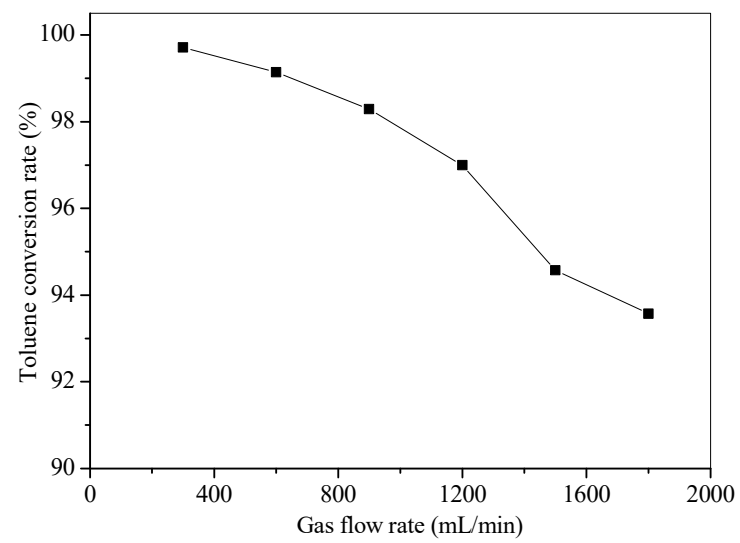

Fig. 6. The relationship between gas flow rate and toluene conversion rate

\subsection{The effect of energy density}

The effect of energy density was investigated. The relationship between energy density and conversion rate of toluene was shown in figure 7 . The energy density has 
serious effect on toluene conversion rate. The conversion rate of toluene was increased with the increase of energy density. The toluene conversion rate was low when the SIE was under $2.0 \mathrm{~kJ} / \mathrm{L}$. The toluene conversion rate was increased rapidly when the SIE continued increased. It reached $90 \%$ when the SIE was $3.0 \mathrm{~kJ} / \mathrm{L}$. It has a maximum of $99.8 \%$ when the SIE is $4.14 \mathrm{~kJ} / \mathrm{L}$. Therefore, the SIE of NTP was chosen above $3.0 \mathrm{~kJ} / \mathrm{L}$.

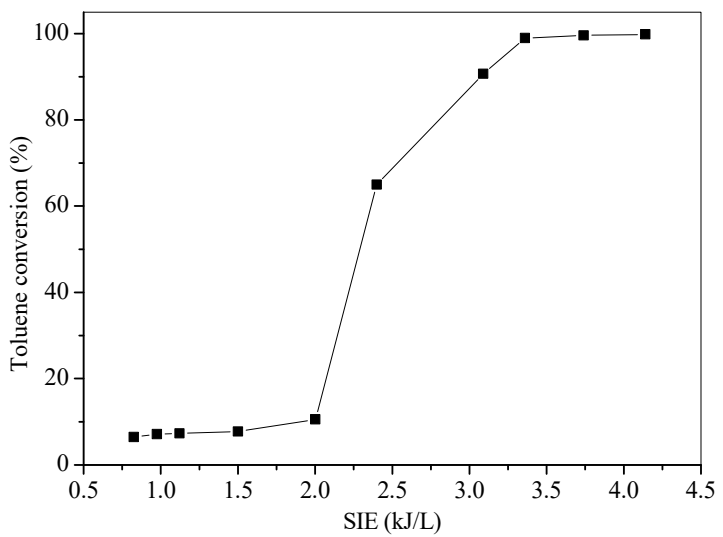

Fig. 7. The relationship between energy density and toluene conversion rate

\subsection{The effect of catalyst assistance}

Table 1 shows the experimental data of toluene removal by catalysts assisted low temperature plasma and single NTP at room temperature with the initial toluene concentration of $700 \mathrm{ppm}$. It can be seen in the table that the toluene concentration rate was improved after catalysis of MO catalysts. The MO derived from the HT showed higher toluene conversion rate than that with single NTP. The reason of this difference is that the MO derived from HT precursor has an effect. The SIE result showed that the MO assisted NTP had higher toluene conversion rate than that of single NTP.

Table 1. Experimental data of toluene removal by catalysts assisted low temperature plasma and single NTP.

\begin{tabular}{|c|c|c|c|}
\hline $\begin{array}{c}\text { Reaction } \\
\text { type }\end{array}$ & $\begin{array}{c}\text { Toluene } \\
\text { concentration } \\
\text { (ppm) }\end{array}$ & $\begin{array}{c}\text { Toluene } \\
\text { conversion } \\
\text { rate (\%) }\end{array}$ & $\begin{array}{c}\text { SIE } \\
(\mathbf{k J} / \mathbf{L})\end{array}$ \\
\hline NTP & 334 & 52.3 & 3.0 \\
\hline $\begin{array}{c}\text { MO assisted } \\
\text { NTP }\end{array}$ & 65 & 90.5 & 3.0 \\
\hline
\end{tabular}

Table 2 shows the experimental data of toluene removal by catalysts assisted low temperature plasma and single NTP with different SIE of NTP. The initial toluene concentration was $700 \mathrm{ppm}$. MO assisted NTP also saved energy when the conversion rate of toluene was the same as shown in table 2. The toluene treatment capacity per unit energy consumption increased from $15.3 \%$ to $20.6 \%$, which had an increase of $34.6 \%$. Thus the HT derived MO assisted NTP showed better effect than the single NTP, which was in correspondence with the literature.

Table 2. Experimental data of toluene removal by catalysts assisted low temperature plasma and single NTP with different SIE.

\begin{tabular}{|c|c|c|c|}
\hline $\begin{array}{c}\text { Reaction } \\
\text { type }\end{array}$ & $\begin{array}{c}\text { Toluene } \\
\text { conversion } \\
\text { rate (\%) }\end{array}$ & $\begin{array}{c}\text { SIE } \\
(\mathbf{k J} / \mathbf{L})\end{array}$ & $\begin{array}{c}\Delta \mathbf{C} / \mathbf{P} \\
(\mathbf{p p m} / \mathbf{W})\end{array}$ \\
\hline NTP & 90.7 & 4.14 & 15.3 \\
\hline $\begin{array}{c}\text { MO assisted } \\
\text { NTP }\end{array}$ & 90.7 & 3.09 & 20.6 \\
\hline
\end{tabular}

\section{Conclusions}

HT precursors were successfully prepared by coprecipitation method and mixed metal oxide catalyst was successfully obtained by calcination of HT precursors. The mixed metal oxide catalyst had high crystalline and good structure. The catalytic performance of $\mathrm{MO}$ catalyst shows that with the increase of reaction time and SIE value of NTP, the conversion rate of toluene was increased. While with the increase of initial concentration of toluene and gas flow rate, the conversion rate of toluene was decreased. With the initial concentration of toluene was $700 \mathrm{ppm}$, the gas flow rate was $600 \mathrm{~mL} / \mathrm{min}$, and the reaction time was 30 min, SIE of NTP was above $3.0 \mathrm{~kJ} / \mathrm{L}$, the toluene conversion rate reached above $90 \%$. Compared with single NTP, MO assisted NTP had better catalytic performance and with lower energy consumption. The toluene treatment capacity per unit energy consumption increased from $15.3 \%$ to $20.6 \%$, which had an increase of $34.6 \%$.

\section{Acknowledgements}

Authors wishing to acknowledge financial support from Guangdong Province Characteristic innovation project of colleges and universities (natural science) "research on catalytic synergistic removal of volatile organic pollutants" (2019).

\section{References}

1. G. Zheng, J. Hou, J. Jiang, J. Fudan Univer. (Natural science edition) 40, 4 (2001)

2. G. Guerra, A. Iemma, D. Lerda, C. Martines, G. Salvi, M. Tamponi. Atmos. Environ. 29, (1995)

3. M Wang. Suzhou Univ., 2016

4. Y. Wu, Zhejing Univ., 2013

5. W. Li, H. Gong. Acta Phys. Chim. Sin., 26, 4(2010) 